\title{
PATHS AND CYCLES IN TOURNAMENTS
}

\author{
ANDREW THOMASON
}

\begin{abstract}
Sufficient conditions are given for the existence of an oriented path with given end vertices in a tournament. As a consequence a conjecture of Rosenfeld is established. This states that if $n$ is large enough, then every non-strongly oriented cycle of order $n$ is contained in every tournament of order $n$.
\end{abstract}

It is well known and easy to see that every tournament has a directed hamilton path. Rosenfeld [8] conjectured that if $n$ is large enough, then any oriented path of order $n$ is contained in any tournament of order $n$. This has been established for alternating paths by Grünbaum [5] and Rosenfeld [8], for paths with two blocks (a block being a maximal directed subpath) by Alspach and Rosenfeld [1] and Straight [10], for paths where the $i$ th block has length at least $i+1$ by Alspach and Rosenfeld [1] and, curiously, for all paths if $n$ is a power of 2 by Forcade [4]. Reid and Wormald [7] have shown that every oriented path of order $n$ is contained in every tournament of order $3 n / 2$.

It is easy to show that a tournament has a strongly oriented hamilton cycle if and only if it is strongly connected. Rosenfeld in [9] conjectured that any non-strongly oriented cycle of order $n$ is contained in any tournament of order $n$, provided $n$ is large enough. This has been verified for cycles with a block of length $n-1$ by Grünbaum, for alternating cycles by Rosenfeld [9] and Thomassen [11], and for cycles with just two blocks by Benhocine and Wojda [2]. (It has also been shown by Heydemann, Sotteau and Thomassen [6] that every digraph of order $n$ with $(n-1)(n-2)+3$ edges contains every non-strong oriented cycle of order $n$.)

In this paper we prove both conjectures (the first is of course a consequence of the second). "Large enough" in this case means at least $2^{128}$, or about $10^{39}$. In fact it seems that the path conjecture is true for $n \geq 8$ (indeed there are probably just three pairs $(P, T)$ with $P \not \subset T)$ and that the cycle conjecture is true for $n \geq 9$. We stress that we make no attempt to give a small lower bound, but aim to establish the conjecture in the shortest time possible. The main result is Theorem 14, which rests on Lemmas 9, 11 and 13. Roughly speaking, Lemma 13 proves the conjecture if the cycle has two separate and fair sized blocks (as do most cycles), Lemma 9 proves it if the cycle has a huge block (as in Grünbaum's result) and Lemma 11 takes care of cycles with many small blocks (such as alternating cycles).

The proof of the conjecture is in the third section. In the first two sections we establish results of independent interest concerning the existence of oriented paths with specified end vertices. Apart from the odd detail, they are as follows: let $P$

Received by the editors August 30, 1982 and, in revised form, January 20, 1985 and July 5, 1985.

1980 Mathematics Subject Classification. Primary 05C20; Secondary 05C45. 
be an oriented path of order $n$ with end blocks of lengths $k$ and $l$, and let $T$ be a tournament containing subsets of vertices $K$ and $L$ with $|K|=k+1$ and $|L|=l+1$. Theorem 1 states that if $T$ has order $n+1$ then we can find $P$ in $T$ with initial vertex in $K$, and Theorem 5 states that if $T$ has order $n+2$ then we can find $P$ in $T$ with initial vertex in $K$ and end vertex in $L$. These theorems are true for all $n$ and are proved by induction. They are best possible in that the values given for $|K|,|L|$ and $|T|$ cannot be reduced. Theorem 1 is proved even though it is largely superseded by Theorem 5, because the proof contains all the ideas of the proof of Theorem 5 but needs much less time spent on small cases. The proof of Theorem 14 (the cycle conjecture) rests heavily on Theorem 5 .

The notation is largely that of Bollobás [3]. If $T$ is a tournament and $u$ is a vertex of $T$ we write $u \in T$ instead of $u \in V(T)$. If $v$ is another vertex of $T$ we indicate the edge directed from $u$ to $v$ by $u v$, and $u v \in T$ is written rather than $u v \in E(T)$. The transitive tournament with vertex set $\left\{x_{1}, \ldots, x_{k}\right\}$ is denoted by $T T_{k}$ or $\left\langle x_{1}, \ldots, x_{k}\right\rangle$, and has all edges $x_{i} x_{j}$ with $i<j$. Note that $T T_{k} \subset T$ if $|T| \geq 2^{k-1}$. If $u \in T$ we write

$$
D_{T}^{+}(u)=\{v \in T ; u v \in T\} \quad \text { and } \quad D_{T}^{-}(u)=\{v \in T ; v u \in T\},
$$

with $d_{T}^{+}(u)=\left|D_{T}^{+}(u)\right|$ and $d_{T}^{-}(u)=\left|D_{T}^{-}(u)\right|$. The subscripts are sometimes omitted. If $X$ and $Y$ are disjoint subsets of $V(T)$ and $x y \in T$ for all $x \in X$ and $y \in Y$ we write $X \Rightarrow Y$ or $Y \Leftarrow X$. If $X=\{x\}$ this becomes $x \Rightarrow Y$ or $Y \Leftarrow x$. Note $x \Rightarrow Y$ implies $x \notin Y$.

Some particular tournaments used are $T C_{3}, T C_{5}$ and $T_{4}^{i}, 1 \leq i \leq 4 . T C_{n}$ has vertices $x_{1}, \ldots, x_{n}$ with $x_{i} x_{i+1} \in T C_{n}$ (addition $\left.\bmod n\right)$ and $x_{i} x_{i+2} \in T C_{5}$ for all $i$. The tournaments $T_{4}^{i}$ are the four tournaments of order four: $T_{4}^{1}$ is $T T_{4}, T_{4}^{2}$ is $x \Rightarrow T C_{3}$ and $T_{4}^{3}$ is $x \Leftarrow T C_{3} . T_{4}^{4}$ is the strong tournament of order 4 , having vertices $a, b, c, d$ and edges $a b, b c, c d, d a, a c$ and $b d$.

Given an oriented path $P$ which starts with $k$ forward edges, followed by $l$ backward edges and $m$ forward edges, we may write $P=P(+k, l, m, \ldots)$. The symbol $P(-k, l, m, \ldots)$ denotes a path beginning with $k$ backward edges, then $l$ forward edges and $m$ backward edges. If the sign is omitted it is assumed positive. $P(k)$ therefore denotes a directed path of order $k+1$ and length $k$; it has one block. The first and last vertices of $P$ are the initial and end vertices.

All tournaments contain a directed hamilton path, and all oriented paths are contained in the transitive tournament of the same order. If $X$ and $Y$ are disjoint subsets of the vertices of a tournament, the notation $X^{+} \rightarrow Y^{+}$denotes a path $P(|X|+|Y|-1)$ consisting of a hamilton path forwards through $X$, an edge to $Y$ and a hamilton path in $Y$. Reversing the signs reverses the hamilton paths; thus $X^{+} \rightarrow Y^{-}$denotes $P(|X|,|Y|-1)$ and $X^{-} \rightarrow Y^{-}$denotes $P(-(|X|-1), 1,|Y|-1)$. The path $X^{+} \rightarrow Y^{+}$may be written $x \rightarrow Y^{+}$if $X=\{x\}$. The expression $u \rightarrow$ $W^{+} \rightarrow v$ does not imply $W \neq \varnothing$, and would denote the edge $u v$ if $W$ were empty. Finally, if $x \in X$ and we wish to specify a hamilton path beginning at $x$ we write $x X^{+}$; likewise $x X^{+} y$ is a path through $X$ from $x$ to $y \in X$. Thus $z X^{+} \leftarrow w$ represents $P(|X|-1,1)$ beginning at $z \in X$ and ending at $w \notin X$.

1. Paths with specified initial vertex. Given an oriented path $P$ and tournament $T$ with $|T| \geq|P|$, it would be helpful to have conditions on a subset $K \subset T$ showing when we may find a copy of $P$ in $T$ whose initial vertex is in $K$. Clearly 
if $K$ is too small this is hopeless; indeed if the length of the first block in $P$ is $k,|K| \leq k$ and $K \Leftarrow(T-K)$, there is no copy of $P$ in $T$ starting in $K$. It turns out that if $|K| \geq k+1$ it is usually possible to find $P$ beginning in $K$, and moreover if $|T| \geq|P|+1$ it is always possible, as the next theorem shows.

THEOREM 1. Let $P$ be an oriented path of order $n$ and first block length $k$. Let $T$ be a tournament of order $n+1$ and let $K$ be a set of at least $k+1$ vertices of $T$. Then there is a copy of $P$ in $T$ with initial vertex in $K$.

ProOF. The proof is by induction on $n$. Let $P=P(k, m, p, \ldots)$. If $P=P(k)$, then the theorem is immediate; this observation provides the base of the induction. From now on we assume $m \geq 1$. Observe that we are done by the induction hypothesis if we can find a path $P^{*}$ in $T$ with initial vertex in $K$ and end vertex of indegree or outdegree (as appropriate) in $T-P^{*}$ at least $k^{\prime}+1$, such that $P$ is equivalent to $P^{*}$ followed by an edge (of the appropriate direction) followed by a path with first block length $k^{\prime}$.

Case (a): $k \geq 2$. Suppose there is a $w \in K$ with $d_{T}^{+}(w) \geq 2$. Then we may choose $X \subset D_{K}^{-}(w)$ with $|X|=\min \left(d_{K}^{-}(w), k-2\right)$ and let $P^{*}=X^{+} \rightarrow w$.

Otherwise $d_{T}^{+}(w) \leq 1$ for every $w \in K$. Then $k=2, K=T C_{3}$ and $K \Leftarrow(T-K)$. By the induction hypothesis $T-K$ contains a copy of $P^{\prime}=P(-(m-1), p, \ldots)$, giving the path $P=K^{+} \leftarrow P^{\prime}$ beginning in $K$.

Case (b): $k=1, m \geq 4$. We may suppose $K$ is the edge $u v$, and let $H=T-K$. Then $|H|=n-1 \geq k+m \geq 5$, so there is a vertex $w \in H$ with $d_{H}^{-}(w) \geq 2$. Let $z$ be the end vertex of a directed hamilton path in $D_{H}^{+}(w)$ (put $z=w$ if $d_{H}^{+}(w)=0$ ); put $\delta=1$ if $z v \in T$ and $\delta=0$ if $v z \in T$. Let $X$ be a subset of $D_{H}^{+}(w)$ containing a path of order $|X|$ ending at $z$, with

$$
|X|=\min \left(d_{H}^{+}(w), m-2-\delta\right) .
$$

Thus we have

$$
\begin{array}{rlr}
\text { either } & P^{*}=u \rightarrow v \leftarrow z X^{-} \leftarrow w & \text { if } z v \in T, \\
\text { or } & P^{*}=v \rightarrow z X^{-} \leftarrow w & \text { if } v z \in T .
\end{array}
$$

Case (c): $k=1, m=3$. Again let $K$ be the edge $u v$ and let $H=T-K$. If $w \in H$ has $d_{H}^{-}(w) \geq 3$, we have $P=v \rightarrow w \leftarrow P(-2, p, \ldots)$ if $v w \in T$ or $P=u \rightarrow v \leftarrow w \leftarrow P(-1, p, \ldots)$ if $w v \in T$. Otherwise $d_{H}^{-}(w) \leq 2$ for all $w \in H$, so $|H| \leq 5, n=5$ or 6 , and $H$ is $T C_{5}, T_{4}^{2}$ or $T_{4}^{4}$. In any of these tournaments we can find at least three vertices which are the initial vertices of copies of $P(-3, p)$, where $p=0$ or 1 as appropriate. If any of these three vertices is in $D_{H}^{+}(v)$ we are home. Otherwise $d_{H}^{-}(v) \geq 3$, and so we get $u \rightarrow v \leftarrow P(-2, p)$.

Case (d): $k=1, m=2$. With $K$ and $H$ as before, we find $u \rightarrow v \leftarrow P(-1, p, \ldots)$ if $d_{H}^{-}(v) \geq 2$ or $v \rightarrow P(-2, p, \ldots)$ if $d_{H}^{+}(v) \geq 3$. If neither of these holds then $d_{H}^{-}(v) \leq 1$ and $d_{H}^{+}(v) \leq 2$, so $|H| \leq 3$. Hence $n=4, P=P(1,2)$, and the result is verified by inspection.

Case (e): $k=m=1$. In this case, if $d_{H}^{+}(v) \geq 2$ we have $v \rightarrow P(-1, p, \ldots)$. Otherwise $d_{H}^{-}(v) \geq n-2 \geq p+1$, giving $u \rightarrow v \leftarrow P(p, \ldots)$.

The following corollary is immediate. 
COROLLARY 2. Every oriented path of order $n$ is contained in every tournament of order $n+1$.

We will later (Theorem 14) give a proof that every oriented path of order $n$ is contained in every tournament of order $n$, provided $n \geq n_{0}$. The value of $n_{0}$ we will give however is large compared to the likely best possible value of $n_{0}$, namely 8. The following assertion, if true, would give this low value for $n_{0}$ :

Given an oriented path $P$ of order $n$ with first block length $k$, a tournament $T$ of order $n$ and $K \subset T,|K|=k+2$, there is a copy of $P$ in $T$ with initial vertex in $K$ unless $n<8$ or $P=P(1, n-2), K=T C_{3}$ and $K \Leftarrow(T-K)$. It should be possible to prove this assertion using the method of proof of Theorem 1 , but this would involve a lot of analysis of small cases which would best be done on a computer.

2. Paths with both ends specified. It is clear from the proof of Theorem 1 that there is an enormous advantage in being able to specify the initial vertex of a path. It is natural to try and go a step further and specify the end vertex also. An obvious extension of Theorem 1 would be to require the path $P$ to have initial vertex in $K$ and end vertex in $L$, where $|L|=l+1$ and $l$ is the length of the final block of $P$. This is achieved in Theorems 3-5 but at the expense of having a further spare vertex in the tournament. Nonetheless Theorems 3-5 will provide the main tool in the proof of the principal result of $\S 3$.

We begin with two theorems dealing with cases not covered by Theorem 5 . The proofs of Theorems 4 and 5 follow very closely that of Theorem 1 .

THEOREM 3. Let $P$ be an oriented path of order $n$ with just two blocks, of lengths $k$ and $l=n-1-k$. Let $T$ be a tournament of order $n+1$ and let $K, L$ be disjoint subsets of $V(T)$ of orders $k+1$ and $l+1$ respectively. Then there is a copy of $P$ with initial vertex in $K$ and end vertex in $L$.

ProOF. Choose a hamilton path in $K$ ending at $u \in K$ and a hamilton path in $L$ ending at $v \in L$. These two paths together with the edge $u v$ or $v u$ contain a suitable copy of $P$.

THEOREM 4. Let $P$ be an oriented path of order $n$ with just three blocks, of lengths $k, 1$ and $l=n-2-k$. Let $T$ be a tournament and let $K, L$ be disjoint subsets of $V(T)$ with $|L| \geq l+1$. Then there is a copy of $P$ in $T$ with initial vertex in $K$ and end vertex in $L$ if

$$
\begin{aligned}
\text { either (i) }|T|=n+1,|K| \geq k+2 \text { and } l \geq 2 \\
\text { or (ii) }|T|=n+2,|K|=k+3 \text { and } l=1
\end{aligned}
$$

PROOF. The proof is by induction on $n$. Let $\delta=0$ if $l \geq 2$ and $\delta=1$ if $l=1$.

Case (a): $k \geq 2$. Suppose there is a vertex $w \in K$ with $d_{K}^{+}(w) \geq 3+\delta$. Choose $X \subset D_{K}^{-}(w)$ with $|X|=\min \left(d_{K}^{-}(w), k-2\right)$. A similar argument to that used in the proof of Theorem 1 shows that, by the induction hypothesis, there is a copy of $P$ beginning with $X^{+} \rightarrow w$ and ending in $L$.

Otherwise every vertex $w \in K$ has $d_{K}^{+}(w) \leq 2+\delta$, and so $d_{K}^{-}(w) \geq k-1$. Since $|K| \geq 4$ we may choose $w \in K$ with $d_{K}^{+}(w) \geq 2$ and $d_{K}^{-}(w) \geq k-1$. Choose $X \subset D_{K}^{-}(w)$ with $|X|=k-1$. By Theorem 3 there is a copy of $P$ beginning with $X^{+} \rightarrow w$ and ending in $L$. 
Case (b): $k=1$. If $|K| \geq 4$ or $K=T T_{3}$ there is a $w \in K$ with $d_{K}^{+}(w) \geq 2$, so by Theorem 3 there is a copy of $P$ beginning at $w$ and ending in $L$.

Otherwise $l \geq 2$ and $K=T C_{3}$. Choose a hamilton path in $L$ and let the first and second vertices of it be $u$ and $v$ respectively. Let the vertices of $K$ be $a, b, c$ with $a b, b c$ and $c a$ in $T$. If $u a \in T$ we have $c \rightarrow a \leftarrow u \rightarrow v P(l-1)$. So we assume $a u \in T$ and likewise $b u, c u \in T$. If $a v \in T$ we get $c \rightarrow u \leftarrow a \rightarrow v P(l-1)$, so we assume $v a \in T$ and likewise $v b, v c \in T$. Finally, either $u x \in T$ for all $x \in L$, in which case we have $c \rightarrow a \leftarrow v \rightarrow b \rightarrow u P(l-2)$, or else $y u \in T$ for some $y \in L$. In the latter case it is easily seen that there is a path $P^{\prime}$ of length $l$ in $L$ beginning at $v$ (or see Lemma 7), giving $P=b \rightarrow c \leftarrow v P^{\prime}$.

The main result of this section now follows.

THEOREM 5. Let $P$ be an oriented path of order $n$ with first block length $k$ and last block length $l$, where $k+l \leq n-3$. Let $T$ be a tournament of order $n+2$ and let $K, L$ be disjoint subsets of $T$ of orders at least $k+1$ and $l+1$ respectively. Then there is a copy of $P$ in $T$ with initial vertex in $K$ and end vertex in $L$.

ProOF. The proof is by induction on $n$. Let $P=P(k, m, \ldots, p, l)$.

Case (a): $k \geq 2$ or $l \geq 2$. Without loss of generality assume $k \geq 2$. Let $H=T-L$. Suppose there is a vertex $w \in K$ with $d_{H}^{+}(w) \geq 2$. Choose $X \subset D_{K}^{-}(w)$ with $|X|=\min \left(k-2, d_{k}^{-}(w)\right)$. By the induction hypothesis there is a copy of $P$ beginning with $X^{+} \rightarrow w$ and ending in $L$.

Otherwise $d_{H}^{+}(w) \leq 1$ for every $w \in K$. Then $K=T C_{3}$ and $K \Leftarrow(T-K-L)$. Again by the induction hypothesis or by Theorems 3 and 4 there is a copy of $P^{\prime}=P(-(m-1), \ldots, p, l)$ in $T^{\prime}=T-K$ beginning in $T^{\prime}-L$ and ending in $L$ (note $P^{\prime}$ has at least two blocks since $k+l \leq n-3$ ). Since $K \Leftarrow\left(T^{\prime}-L\right.$ ) we have the copy $K^{+} \leftarrow P^{\prime}$ of $P$ we desire.

For the remaining cases, in which $k=l=1$, we assume $K=u v, L=x y$ and $H=T-K-L$.

Case (b): $k=l=1, m \geq 4$. Let $K=u v$, and choose $w \in H$ with $d_{H}^{-}(w) \geq 2$; this can be done as $|H|=n-k-l \geq m+1 \geq 5$. Pick a hamilton path in $D_{H}^{+}(w)$ and let $z$ be its end vertex. (Put $z=w$ if $d_{H}^{+}(w)=0$.) Let $X$ be the set of the last $\min \left(m-2-\delta, d_{H}^{+}(w)\right)$ vertices of this path, where $\delta=1$ if $z v \in T$ and $\delta=0$ if $v z \in T$. Observe that $z \in X$ if $X \neq \varnothing$. Then by Theorem 3 or by the induction hypothesis there is a copy of $P$ ending in $L$ and beginning

$$
\begin{array}{ll}
u \rightarrow v \leftarrow z X^{-} \leftarrow w & \text { if } z v \in T, \\
\text { or } \quad v \rightarrow z X^{-} \leftarrow w & \text { if } v z \in T,
\end{array}
$$

unless $P=P(1, m, 1,1)$. This case is identical to the case $P=P(1,1, p, 1)$ and is covered by Case (c).

Case (c): $k=l=1, m \leq 3, n \geq 7$. Suppose $m \geq 2$, and consider first the case $P \neq P(1, m, 1,1)$. If $d_{H}^{+}(v) \geq m+1$ or if $d_{H}^{-}(v) \geq m$, arguments of a by now familiar kind show we are home. Otherwise $|H|=n-2 \leq 2 m-1 \leq 5$, so $n=7$ and $m=3$. But this means $P=P(1,3,1,1)$. So suppose now $P=P(1, m, 1,1)$. We are again home if $d_{H}^{+}(y) \geq 2$ or $d_{H}^{-}(y) \geq m+1$. But one of these must happen, for otherwise $|H|=n-2 \leq m+1 \leq 4$. Thus the case $m \geq 2$ is taken care of, and $p \geq 2$ is dealt with similarly. 
This leaves $P=P(1,1, s, \ldots, 1,1)$, where $s \geq 1$. If $d_{H}^{+}(v) \geq 2$ we are home by induction, so we may assume $d_{H}^{+}(v) \leq 1$ and hence $d_{H}^{-}(v) \geq n-3$. Here again we are home by induction unless $P=P(1,1, n-5,1,1)$. But then either $d_{H}^{-}(x) \geq 2$, in which case we may apply induction, or $d_{H}^{+}(x) \geq n-3$, when choosing $X \subset D_{H}^{-}(v) \cap D_{H}^{+}(x)$ with $|X|=n-4$ gives $P=u \rightarrow v \leftarrow X^{+} \leftarrow x \rightarrow y$.

Case (d): $k=l=1, n \leq 6$. The cases $P=P(1,2,1,1)$ and $P=P(1,1,1,1,1)$ were in fact covered by the proof of Case (c). This leaves three possibilities. Since the whole proof is based on induction the small cases are not insignificant, and we outline them here.

$P=P(1,3,1)$ : Suppose $w \in H$ has $d_{H}^{-}(w) \geq 2$. Then by Theorem 3 we can find $P^{\prime}=P(-1,1)$ from $D_{H}^{-}(w)$ to $L$, giving $P=u \rightarrow v \leftarrow w \leftarrow P^{\prime}$ unless $v w \in T$. If further $d_{H}^{-}(w)=3$ we now find $P^{\prime}=P(-2,1)$ from $D_{H}^{-}(w)$ to $L$, giving $P=v \rightarrow w \leftarrow P^{\prime}$ from $K$ to $L$. Thus we may suppose $d_{H}^{-}(w) \leq 2$ for all $w \in H$, and that $d_{H}^{-}(w)=2$ implies $v w \in T$. Due to the symmetry of $P$, a similar argument shows we may suppose $d_{H}^{+}(w) \leq 2$ for all $w \in H$, and that $d_{H}^{+}(w)=2$ implies $w x \in T$ (where $L=x y$ ). But then $H=T_{4}^{4}$ and the four implied edges yield $P(1,3,1)$ from $v$ to $x$, as desired.

$P=P(1,2,1)$ : As usual we may assume $d_{H}^{+}(v)=2, d_{H}^{-}(v)=1$, and likewise $d_{H}^{-}(x)=2, d_{H}^{+}(x)=1$, where $L=x y$. Let $H$ have vertices $a, b$, and $c$ with $a b, b c \in H$. If both $v c$ and $a x$ are in $T$, we have $v \rightarrow c \leftarrow b \leftarrow a \rightarrow x$. Otherwise by symmetry we may assume $c v \in T$, so $v a, v b \in T$ since $d_{H}^{+}(v)=2$. Then $c x \in T$ (else we get $u \rightarrow v \leftarrow c \leftarrow x \rightarrow y$ ) and $a c \in T$ (else we have $v \rightarrow b \leftarrow a \leftarrow c \rightarrow x$ ). Finally either $a x \in T$, giving $u \rightarrow v \leftarrow c \leftarrow a \rightarrow x$, or $x a \in T$, giving $v \rightarrow b \leftarrow a \leftarrow x \rightarrow y$.

$P=P(1,1,1,1)$ : We may suppose $d_{H}^{-}(v) \leq 1$ and likewise $d_{H}^{-}(y) \leq 1$. Let $H$ have vertices $a, b$ and $c$.

If $d_{H}^{+}(v)=3$ and $H=T T_{3}$, then $d_{H}^{-}(a)=2$, say, and we may find $P^{\prime}=P(1,1)$ from $\{b, c\}$ to $\{x, y\}$ giving $v \rightarrow a \leftarrow P^{\prime}$ from $K$ to $L$. Hence if $d_{H}^{+}(v)=3$ we may assume $H=T C_{3}$, with say $a b, b c$ and $c a$ in $H$. Since $a y \in T$ gives $v \rightarrow b \leftarrow a \rightarrow y \leftarrow x$ we may assume $y a \in T$, and likewise $y b, y c \in T$. By symmetry we may now suppose $u x \in T$; then either $x a \in T$ giving $v \rightarrow c \leftarrow y \rightarrow a \leftarrow x$, or $a x \in T$, giving $u \rightarrow x \leftarrow a \rightarrow b \leftarrow y$.

Otherwise $d_{H}^{+}(v)=2$, and likewise we may assume $d_{H}^{+}(y)=2$. If $D_{H}^{-}(v) \cap$ $D_{H}^{-}(y) \neq \varnothing$ we are home, so we assume $a v, y a, v b, y b, v c$ and $c y$ are in $T$. Now if $a b \in T$ we get $u \rightarrow v \leftarrow a \rightarrow b \leftarrow y$, so assume $b a \in T$. Then either $c b \in T$, giving $v \rightarrow b \leftarrow c \rightarrow y \leftarrow x$, or $b c \in T$, giving $v \rightarrow c \leftarrow b \rightarrow a \leftarrow y$.

Having proved Theorem 5 , we are able to easily deduce the next corollary.

COROLLARY 6. Let $C$ be a non-strongly oriented cycle of order $n$, and let $T$ be a tournament of order $n+2$. Then $T$ contains $C$ provided $n \geq 14$.

Proof. Suppose $C$ has a block of length $b \geq 4$. Find a vertex $w$ with $d_{T}^{+}(w) \geq 4$ and $d_{T}^{-}(w) \geq 2$ (possible as $|T|>10$ ). Now choose $K \subset D_{T}^{+}(w)$ and $L \subset D_{T}^{-}(w)$ with $|K| \geq 4,|L| \geq 2$ and $|K|+|L|=b+2$. (Note $b \leq n-1$.) Then if $P=$ $P(|K|-3, \ldots,|L|-1)$ is a path obtained by removing a suitable vertex from the block of $C$, we may find $P$ in $T-w$ going from $K$ to $L$ (using Theorems 4 and 5), and hence find $C$ in $T$.

Otherwise all blocks of $C$ have length at most 3. Let $u$ be a vertex of $C$ with $d_{C}^{-}(u)=0$ and consider $C-u=P(k, \ldots, l)$, where $k, l \leq 3$. Note $P \neq P(k, 1, l)$ 
since $n>9$. Choose $w \in T$ with $d_{T}^{+}(w) \geq 8$, and choose $K, L$ disjoint in $D_{T}^{+}(w)$ with $|K|=k+1$ and $|L|=l+1$. By Theorem 5 there is a copy of $P(k, \ldots, l)$ in $T-w$ running from $K$ to $L$, which with $w$ gives a copy of $C$ in $T$.

Corollary 6 is probably true for all $n \geq 3$.

3. Cycles. This section contains a proof of the conjecture mentioned in the abstract. The main tool is of course Theorem 5 of $\S 2$, and the work here is devoted to removing the two unused vertices of that theorem.

We will need seven lemmas, of which Lemmas 9, 11 and 13 are the ones which will be used in the proof of the main result, Theorem 14. The first lemma is a straightforward observation.

LEMMA 7. Let $P=x_{1} \rightarrow x_{2} \rightarrow \cdots \rightarrow x_{k}$ be a path in a tournament $T$, and let $Y \subset T$ be a set of vertices such that for each $y \in Y$ there is an $x_{i}$ with $x_{i} y \in T$. Then there is a directed hamilton path in $P \cup Y$ beginning at $x_{1}$. If further for each $y \in Y$ we can find $i<j$ such that $x_{i} y, y x_{j} \in T$, then there is a directed hamilton path in $P \cup Y$ beginning at $x_{1}$ and ending at $x_{k}$.

PROOF. It is enough to prove the lemma when $|Y|=1$ provided the path we construct preserves the order of the $x_{i}$ 's. Suppose then $Y=\{y\}$. Then either there is some $i$ with $x_{i} y, y x_{i+1} \in T$, when $x_{1} \rightarrow x_{2} \rightarrow \cdots \rightarrow x_{i} \rightarrow y \rightarrow x_{i+1} \rightarrow \cdots \rightarrow x_{k}$ is a suitable path, or else $x_{k} y \in T$, when $x_{1} \rightarrow x_{2} \rightarrow \cdots \rightarrow x_{k} \rightarrow y$ is a suitable path.

LEMMA 8. Let $S$ be a transitive subtournament of a tournament $T$, with $|S| \geq$ 4. Let $X=T-S$ and suppose that for every $x \in X$ there are $y, z \in S$ with $x y, z x \in T$. Let $m$ be an integer with $|X|+3 \leq m \leq|T|-1$. Then there is an edge $u v$ in $S$ and a directed path $P$ of length $m$ from $u$ to $v$ which contains $X$, such that $u \Rightarrow(T-P)$ and $v \Leftarrow(T-P)$.

PROOF. Let $S=\left\langle w_{1}, \ldots, w_{k}\right\rangle$. Let $Y \subset X$ be maximal such that $Y$ contains a directed hamilton path, say $P=x_{1} \rightarrow \cdots \rightarrow x_{r}$ (where $r=|Y|$ ), with $w_{i} x_{1}$ and $x_{r} w_{j}$ in $T$ for distinct $w_{i}$ and $w_{j}$. Note $Y \neq \varnothing$ unless $X=\varnothing$ when the lemma is trivial. If $x \in X-Y$, then $Y \cup\{x\}$ has by Lemma 7 a directed hamilton path whose end vertices are either $x_{1}$ and $x_{r}, x$ and $x_{r}$ or $x_{1}$ and $x$. By the maximality of $Y$ the first possibility cannot occur, and the others imply either $D_{S}^{-}(x)=\left\{w_{j}\right\}$ or $D_{S}^{+}(x)=\left\{w_{i}\right\}$. Hence $X-Y=X_{1} \cup X_{2}$, where

$$
X_{1}=\left\{x \in X-Y ; D_{S}^{+}(x)=\left\{w_{i}\right\}\right\} \quad \text { and } \quad X_{2}=\left\{x \in X-Y ; D_{S}^{-}(x)=\left\{w_{j}\right\}\right\} .
$$

Let $u$ be the vertex of $S-\left\{w_{i}, w_{j}\right\}$ with the smallest subscript, unless $X_{1}=\varnothing$, when $u$ is the vertex of $S-\left\{w_{j}\right\}$ with the smallest subscript. Let $v$ be the vertex of $S-\left\{w_{i}, w_{j}\right\}$ with the largest subscript, unless $X_{2}=\varnothing$, when $v$ is the vertex of $S-\left\{w_{i}\right\}$ with the largest subscript. Then

$$
Q=u \rightarrow X_{1}^{+} \rightarrow w_{i} \rightarrow P \rightarrow w_{j} \rightarrow X_{2}^{+} \rightarrow v
$$

is a path from $u$ to $v$ containing $X$, with $u \Rightarrow(T-Q), v \Leftarrow(T-Q)$ and $u v \in T$. Note $Q$ has length at most $|X|+3$. To obtain $P$, let $M$ be $m+1-|Q|$ vertices of $T-Q$. Since $u \Rightarrow M$ and $M \Rightarrow v$ there is by Lemma 7 a directed hamilton path $P$ in $Q \cup M$ from $u$ to $v$. This path $P$ satisfies the requirements of the lemma. 
LEMMA 9. Let $C$ be a non-strongly oriented cycle of order $n$, and let $T$ be a tournament of order $n$. If $C$ has a block of length $b$ and $T$ has a transitive subtournament of order $n-b+3$, then $T$ contains $C$.

PROOF. Let $S$ be a maximal transitive subtournament of order at least $n-b+3$. Then $|S| \geq 4$, and if $X=T-S$ then $X, T$ and $S$ satisfy the conditions of Lemma 8 (since $S$ is maximal). Now $|X|+3=n-|S|+3 \leq b$, so by Lemma 8 we can find $u, v \in S$ with a path $P$ of length $b$ from $u$ to $v$, with $u \Rightarrow(T-P), v \Leftarrow(T-P)$ and $u v \in T$. Since $T-P$ is transitive it contains the remainder of $C$, and hence $T$ contains $C$.

Note that Lemma 9 provides a proof of Grünbaum's result, where $C$ has exactly two blocks of lengths 1 and $n-1$.

LEMMA 10. Let $P$ be an oriented path with at least 40 vertices and at least 3 blocks, and let $T$ be a tournament of order $|P|$. Suppose that $T$ contains a transitive subtournament $S$ of order $|T|-2$. Then $T$ contains a copy of $P$ with initial and end vertices in $S$.

ProOF. Suppose first that $P$ has a block of length $b \geq 5$. Let $T-S=\left\{x_{1}, x_{2}\right\}$ and let $X$ be the maximal subset of $\left\{x_{1}, x_{2}\right\}$ such that $X$ and $S$ satisfy the conditions of Lemma 8 . If $|X|=2$, we may by Lemma 8 find a path $P_{1}$ of length $b$ with ends $u$ and $v$ such that $T-P_{1} \subset S$. Since $T-P_{1}$ is transitive it contains $P-P_{1}$ and this gives us $P$, because $u \Rightarrow\left(T-P_{1}\right) \Rightarrow v$. If $X=\left\{x_{2}\right\}$, say, we may suppose $x_{1} \Leftarrow S$. We may also write $P$ as $R \rightarrow x \leftarrow Q$ where $R$ and $Q$ are paths and $R$, say, contains a block of length at least $b-1$. We may then find a copy $R^{\prime}$ of $R$ containing $X$ with its ends in $S$, by a similar argument to the above. But now $R^{\prime} \rightarrow x_{1} \Leftarrow(S-R)$ contains $P$, since $Q \subset(S-R)$. If $X=\varnothing$ the lemma is straightforward.

Otherwise every block of $P$ has length at most 4 . We may suppose that $P$ can be written $Q_{1} \rightarrow Q_{2}$, where $Q_{1}$ and $Q_{2}$ are oriented paths of lengths 19 and $|P|-21$ respectively. Let $S=\left\langle w_{1}, \ldots, w_{|S|}\right\rangle$. We show that $Q_{1}$ can be found in $\left\langle w_{1}, \ldots, w_{19}\right\rangle \cup\left\{x_{1}\right\}$ with its ends in $\left\langle w_{1}, \ldots, w_{19}\right\rangle$. Similarly $Q_{2}$ can be found in $\left\langle w_{20}, \ldots, w_{|S|}\right\rangle \cup\left\{x_{2}\right\}$, which gives $P$. To see that $Q_{1}$ can be found as claimed, observe that two of the edges between $x_{1}$ and $\left\{w_{9}, w_{10}, w_{11}\right\}$ have the same direction; say without loss of generality that $w_{10} x_{1}, w_{11} x_{1} \in T$. Write $Q_{1}=R_{1} \rightarrow x \leftarrow R_{2}$, where neither $R_{1}$ nor $R_{2}$ are devoid of edges, and let $B_{1}$ (resp. $B_{2}$ ) be the last (resp. first) block of $R_{1}$ (resp. $R_{2}$ ). By taking two suitable disjoint paths of length at most 4 from $\left\{w_{10}, w_{11}\right\}$ to $\left\{w_{1}, w_{2}, w_{18}, w_{19}\right\}$ we can construct $B_{1} \rightarrow x \leftarrow B_{2}$, and since the ends of this path are at the extreme vertices of $\left\langle w_{1}, \ldots, w_{19}\right\rangle$ we get the rest of $Q_{1}$.

LEMMA 11. Let $C$ be a non-strongly oriented cycle of order $n$ containing a set $E$ of $q \geq 43$ consecutive edges, such that $E$ is a union of blocks of $C$ and such that the $q-6$ consecutive edges obtained by deleting the first three and last three edges of $E$ contain at least one block of $C$. Let $T$ be a tournament of order $n$ containing a transitive subtournament of order $q+1$. Then $T$ contains $C$.

ProOF. Let $S$ be a transitive subtournament of order $q+1$, say $S=\left\langle w_{0}, \ldots, w_{q}\right\rangle$. We may suppose the second edge of $E$ goes from the second vertex to the third. Let $K=\left\{w_{0}, w_{1}, w_{2}, w_{3}\right\}$ and let $L=\left\{w_{q-1}, w_{q}\right\}$ or $L=\left\{w_{4}, w_{5}\right\}$ according as the penultimate edge of $E$ has the same or opposite direction as the second. Let 
$E_{1}$ be $E$ with the first and last edges removed, and let $E_{2}$ be $E$ with the first two and last two edges removed. Let $P_{1}$ be the path obtained from $C$ by removing $E_{1}$, and let $P_{2}$ be the path containing the edges of $E_{2}$. Then by the conditions on $E, P_{1}=P( \pm 1, \ldots, 1)$ and $P_{2}$ has $q-3 \geq 40$ vertices and at least three blocks. By Theorems 3-5 we may find $P_{1}$ in $T_{1}=(T-S) \cup K \cup L$ with its ends in $K$ and $L$, and to form $C$ we need only find $P_{2}$ in $T-P_{1}$ with its ends in $S-K-L$. But we can achieve this by Lemma 10 , because $T-P_{1}=(S-K-L) \cup\left(T_{1}-P_{1}\right)$, where $S-K-L$ is transitive of order $\left|P_{2}\right|-2$ and $\left|T_{1}-P_{1}\right|=2$.

Observe that Lemma 11 covers the case of alternating cycles.

We now have lemmas showing $C \subset T$ if $C$ contains many small blocks or if $C$ contains a very large block. Many pairs $(C, T)$ still remain unaccounted for, but are covered by Lemma 13, which is itself based on the next lemma.

LEMMA 12. Let $S$ be a maximal transitive subtournament of a tournament $T$. Let $X, Y, Z$ be pairwise disjoint subsets of $T-S$, and let $P=P(k, \ldots, l)$ be a path of order $|S|+|X|+|Y|+|Z|$ with at least two blocks, such that $k \geq|X|+|Z|+6$ and $l \geq|Y|+|Z|+6$. Suppose there are vertices $x, y \in S$ such that $x \Rightarrow X \cup(S-\{x\})$ and such that $y \Rightarrow Y \cup(S-\{x, y\})$ or $y \Leftarrow Y \cup(S-\{x, y\})$ according as $P$ has an even or an odd number of blocks. Then there is a copy of $P$ in $S \cup X \cup Y \cup Z$ with initial vertex $x$ and end vertex $y$.

ProOF. Notice that since $|S|+|X|+|Y|+|Z| \geq k+l+1$ we have $|S| \geq 13$. Let $S_{1}=S-\{x, y\}$, let $Y^{\prime}=\left\{z \in Z ; z \Rightarrow S_{1}\right\}$ and let $X^{\prime}=Z-Y^{\prime}$. Put $X \cup X^{\prime}=X_{1} \cup X_{2} \cup X_{3}$, where

$$
X_{1}=\left\{w \in X \cup X^{\prime} ; w \Rightarrow S_{1}\right\}, \quad X_{3}=\left\{w \in X \cup X^{\prime} ; w \Leftarrow S_{1}\right\}
$$

and

$$
X_{2}=\left(X \cup X^{\prime}\right)-X_{1}-X_{3} .
$$

Observe that $X^{\prime} \subset X_{2} \cup X_{3}$, so $x \Rightarrow X_{1}$. By Lemma 8 there are vertices $u_{1}$ and $v_{1}$ in $S_{1}$ and a path $P_{1}$ in $S_{1} \cup X_{2}$ of order $\left|X_{2}\right|+4$ from $u_{1}$ to $v_{1}$, containing $X_{2}$ and such that $u_{1} \Rightarrow S_{2}, v_{1} \Leftarrow S_{2}$ and $u v \in T$, where $S_{2}=\left(S_{1} \cup X_{2}\right)-P_{1}$. This gives a path

$$
x \rightarrow X_{1}^{+} \rightarrow u_{1} P_{1} v_{1} \rightarrow X_{3}^{+} \Leftarrow S_{2},
$$

whose first block is of length $\left|X \cup X^{\prime}\right|+4 \leq|X|+|Z|+4$. Note this path works even if some of the $X_{i}$ 's are empty, since $x u_{1} \in T$ and $v_{1} \Leftarrow S_{2}$.

If $P$ has an even number of blocks, consider some $z \in Y^{\prime}$. If $z y \in T$, then either $\langle x, z, y, \ldots\rangle$ or $\langle z, x, y, \ldots\rangle$ is a transitive tournament containing $S$. Hence by the maximality of $S, y z \in T$, and so $y \Rightarrow Y^{\prime}$. Thus we can repeat the argument of the previous paragraph with $x, X$ and $S_{1}$ replaced by $y, Y$ and $S_{2}$, and since $y \Rightarrow Y_{1}$ we have the path

$$
y \rightarrow Y_{1}^{+} \rightarrow u_{2} P_{2} v_{2} \rightarrow Y_{3}^{+} \Leftarrow S_{3},
$$

where $S_{3}=\left(S_{2} \cup Y_{2}\right)-P_{2}$. The first block of this path has length $\left|Y \cup Y^{\prime}\right|+4 \leq$ $|Y|+|Z|+4$, and furthermore $\left|S_{3}\right|=\left|S_{1}\right|-8=|S|-10$.

On the other hand, if $P$ has an odd number of blocks, then partition $Y \cup Y^{\prime}$ as $Y_{1} \cup Y_{2} \cup Y_{3}$, where

$$
Y_{1}=\left\{w \in Y \cup Y^{\prime} ; w \Leftarrow S_{2}\right\}, \quad Y_{3}=\left\{w \in Y \cup Y^{\prime} ; w \Rightarrow S_{2}\right\}
$$


and

$$
Y_{2}=\left(Y \cup Y^{\prime}\right)-Y_{1}-Y_{3}
$$

Since $Y^{\prime} \subset Y_{3}$ we have $y \Leftarrow Y_{1}$, and so in a similar manner a path

$$
S_{3} \Leftarrow Y_{3}^{+} \rightarrow u_{2} P_{2} v_{2} \rightarrow Y_{1}^{+} \rightarrow y
$$

can be constructed, the last block being of length $\left|Y \cup Y^{\prime}\right|+4$. Notice $\left|S_{3}\right|=$ $|S|-10>0$.

Suppose first that $|P| \geq k+l+3$. Choose $R_{1} \subset S_{3}$ with $\left|R_{1}\right|=k-\left|X \cup X^{\prime}\right|-4$ and $R_{2} \subset S_{3}-R_{1}$ with $\left|R_{2}\right|=l-\left|Y \cup Y^{\prime}\right|-4$. Let $R=S_{3}-R_{1}-R_{2}$. Note $|R|=\left|S_{3}\right|-\left|R_{1}\right|-\left|R_{2}\right|=|P|-2-k-l \geq 1$. By Lemma $7, u_{i} P_{i} v_{i}$ together with $R_{i}$ contains a directed hamilton path $Q_{i}$ from $u_{i}$ to $v_{i}$, because $u_{i} \Rightarrow R_{i} \Rightarrow v_{i}$. We now have

$$
x \rightarrow X_{1}^{+} \rightarrow Q_{1}^{+} \rightarrow X_{3}^{+} \Leftarrow R \Rightarrow Y_{3}^{-} \leftarrow Q_{2}^{-} \leftarrow Y_{1}^{-} \leftarrow y,
$$

or

$$
x \rightarrow X_{1}^{+} \rightarrow Q_{1}^{+} \rightarrow X_{3}^{+} \Leftarrow R \Leftarrow Y_{3}^{+} \rightarrow Q_{2}^{+} \rightarrow Y_{1}^{+} \rightarrow y,
$$

as appropriate, from $x$ to $y$, with first and last blocks lengths $k$ and $l$ respectively. Since $R$ is transitive, we have $P$ from $x$ to $y$.

Otherwise $|P| \leq k+l+2$, so $P=P(k, 1, l)$ or $P=P(k, l)$. Suppose $P=$ $P(k, 1, l)$. Consider the edge from the end of the directed path $v_{1} \rightarrow X_{3}^{+}$to the beginning of the directed path $Y_{3}^{+} \rightarrow u_{2}$. If this goes from the latter to the former (in particular if $Y_{3}=\varnothing$, since $u_{2} \in S_{2}$ and both $v_{1} \Leftarrow S_{2}$ and $X_{3} \Leftarrow S_{2}$ ) form $Q_{1}$ and $Q_{2}$ as before to get

$$
x \rightarrow X_{1}^{+} \rightarrow Q_{1}^{+} \rightarrow X_{3}^{+} \leftarrow Y_{3}^{+} \rightarrow Q_{2}^{+} \rightarrow Y_{1}^{+} \rightarrow y,
$$

which is the desired $P(k, 1, l)$ from $x$ to $y$. Otherwise this edge goes from $v_{1} \rightarrow X_{3}^{+}$ to a vertex $w \in Y_{3} \neq \varnothing$. Now choose $R_{1} \subset S_{3}$ with $\left|R_{1}\right|=k-\left|X \cup X^{\prime}\right|-6$ and $R_{2} \subset S_{3}-R_{1}$ with $\left|R_{2}\right|=l-\left|Y \cup Y^{\prime}\right|-3$. Form $Q_{1}$ and $Q_{2}$ as before. Notice that

$$
\left|S_{3}-R_{1}-R_{2}\right|=|S|-|P|+|X|+|Y|+|Z|+1=1,
$$

so put $S_{3}-R_{1}-R_{2}=\{r\}$; note $w r \in T$ since $w \in Y_{3}$. We then have

$$
x \rightarrow X_{1}^{+} \rightarrow Q_{1}^{+} \rightarrow X_{3}^{+} \rightarrow w \rightarrow r \leftarrow\left(Y_{3}-w\right)^{+} \rightarrow Q_{2}^{+} \rightarrow Y_{1}^{+} \rightarrow y,
$$

which is the $P(k, 1, l)$ we want.

Finally, suppose $P$ has exactly two blocks; then $|S|+|X|+|Y|+|Z|=k+l+1$. Consider the connecting edge between the end vertices of the paths $v_{1} \rightarrow X_{3}^{+}$and $v_{2} \rightarrow Y_{3}^{+}$; by symmetry we may suppose it goes from the latter to the former. Then choose $R_{1}$ and $R_{2}$ as before but with $\left|R_{1}\right|=k-\left|X \cup X^{\prime}\right|-4$ and $\left|R_{2}\right|=l-\left|Y \cup Y^{\prime}\right|-5$. (Of course, if the connecting edge goes the other way we reduce $R_{1}$ instead of $R_{2}$.) Notice now $R=\varnothing$. Constructing $Q_{1}$ and $Q_{2}$ as before gives

$$
x \rightarrow X_{1}^{+} \rightarrow Q_{1}^{+} \rightarrow X_{3}^{+} \leftarrow Y_{3}^{-} \leftarrow Q_{2}^{-} \leftarrow Y_{1}^{-} \leftarrow y,
$$

which is $P(k, l)$ from $x$ to $y$.

We are now ready to prove the main lemma. 
LEMMA 13. Let $C$ be a non-strongly oriented cycle of order $n$, let $T$ be a tournament of order $n$, and let $S$ be a maximal transitive subtournament of $T$. Suppose that $C$ contains distinct blocks $B_{1}$ and $B_{2}$ of lengths $b_{1}$ and $b_{2}$ each at least 18 , such that the distance $d$ between $B_{1}$ and $B_{2}$ (measured one of the two ways on $C$ ) satisfies $d \leq|S|-33$ and $d+b_{1}+b_{2} \geq|S|+10$. (We allow $d=0$ if $B_{1}$ and $B_{2}$ are adjacent.) Then $T$ contains $C$.

Proof. Let $P_{1}=P(m, \ldots)$ be the path of length $d$ in $C$ from $B_{1}$ to $B_{2}$, and let $P_{2}=P(q, \ldots)$ be the path of length $n-d-b_{1}-b_{2}$ from $B_{1}$ to $B_{2}$. (We may assume, by reversing all arcs in $C$ and $T$ if necessary, that both $m \geq 0$ and $q \geq 0$; either or both of $P_{1}$ and $P_{2}$ may have length zero.) Let $x, y$ be vertices of $S$ defined by $S=\langle x, \ldots, y\rangle$ if $B_{1}$ and $B_{2}$ have the same direction on $C$ (that is, $P_{1}$ and $P_{2}$ have an odd number of blocks) and $S=\langle x, y, \ldots\rangle$ otherwise. Let $H=T-S$. The aim of the proof is to find a path from $x$ to $y$ via $H$ containing as much of $P_{2}, B_{1}$ and $B_{2}$ as possible (using Theorems $3-5$ ), and then to find the rest of $C$ as a path from $x$ to $y$ in $S$ and the other remaining vertices (using Lemma 12).

The proof falls into three cases depending on the values of $d_{H}^{+}(x)$ and $d_{H}^{+}(y)$. In what follows the symbols $d^{1}$ and $d^{2}$ are equivalent to $d^{+}$and $d^{-}$respectively if $B_{1}$ and $B_{2}$ have the same direction in $C$, and equivalent to $d^{-}$and $d^{+}$respectively if $B_{1}$ and $B_{2}$ have opposite directions.

Case $(\mathrm{a}): d_{H}^{-}(x) \geq 6, d_{H}^{1}(y) \geq 6$. Suppose there are integers $k$ and $l$ such that we can find paths $P\left(b_{1}-k, m, \ldots, b_{2}-l\right)$ from $x$ to $y$ and $P(-k, q, \ldots, l)$ from $x$ to $y$ whose intersection is just $\{x, y\}$. Then we will have the desired copy of $C$. To find such paths, first choose $k$ and $l$ by the following procedure (which essentially finds the largest feasible values for $k$ and $l$ ).

Choose $K_{1} \subset D_{H}^{-}(x),\left|K_{1}\right|=4$, and $L_{1} \subset D_{H}^{1}(y)-K_{1},\left|L_{1}\right|=2$. Now choose $K_{2} \subset D_{H}^{-}(x)-K_{1}-L_{1}$ and $L_{2} \subset D_{H}^{1}(y)-K_{1}-L_{1}$ with $K_{2} \cap L_{2}=\varnothing$ and $\left|K_{2}\right|+\left|L_{2}\right|$ as large as possible, subject to
(i) $\left|K_{2}\right| \leq b_{1}-18$,
(ii) $\left|L_{2}\right| \leq b_{2}-18$, and
(iii) $\left|K_{2}\right|+\left|L_{2}\right| \leq d+b_{1}+b_{2}-|S|-5$.

Put $K=K_{1} \cup K_{2}, L=L_{1} \cup L_{2}, k=|K|-2$ and $l=|L|$. Then $k \geq 2$ and $l \geq 2$, and (i)-(iii) are equivalent to

(i) $k \leq b_{1}-16$,

(ii) $l \leq b_{2}-16$, and

(iii) $k+l \leq d+b_{1}+b_{2}-|S|-1$.

Inequality (iii) is the same as

$$
k+l \leq n-|P(q, \ldots)|-|S|,
$$

whence $|P(-(k-1), q, \ldots, l-1)| \leq n-|S|-2=|H|-2$. Let $P^{*}=P(-(k-1)$, $q, \ldots, l-1)$. If $P^{*} \neq P(-(k-1), l-1)$, then $\left|P^{*}\right| \geq k+l$ and we may choose a tournament $T^{*} \subset H$ of order $\left|P^{*}\right|+2$ containing $K$ and $L$; otherwise if $P^{*}=$ $P(-(k-1), l-1)$ put $T^{*}=K \cup L$, whence $T^{*}$ has order $\left|P^{*}\right|+3$.

By Theorems 3-5 we may find a copy of $P^{*}$ in $T^{*}$ beginning in $K$ and ending in $L$. Since $K \subset D_{H}^{-}(x)$ and $L \subset D_{H}^{1}(y)$, this gives a copy of $P(-k, q, \ldots, l)$ from $x$ to $y$. What remains to be done is to find a copy of $P\left(b_{1}-k, m, \ldots, b_{2}-l\right)$ from $x$ to $y$ in $T-P^{*}$. Let $Z=T^{*}-P^{*} ;$ then $2 \leq|Z| \leq 3$. Let $W=T-T^{*}-S$; thus $T-P^{*}=S \cup Z \cup W$. Observe $|W|+|Z|=|T|-\left|P^{*}\right|-|S|=d+b_{1}+b_{2}+1-k-l-|S|$. 
We shall now find a partition of $W$ into $X \cup Y$, with $X \subset D^{+}(x), Y \subset D^{2}(y), b_{1}-$ $k \geq|X|+|Z|+6$ and $b_{2}-l \geq|Y|+|Z|+6$. Then Lemma 12 shows that the desired copy of $P\left(b_{1}-k, m, \ldots, b_{2}-l\right)$ exists.

Suppose first equality holds in (iii). Then $|W|+|Z|=2$, so $|Z|=2$ and $W=$ $X=Y=\varnothing$. Note $b_{1}-k \geq 16 \geq|Z|+6, b_{2}-l \geq 16 \geq|Z|+6$. Now suppose that inequality (iii) is strict. Observe that strict inequality in (i) now means that $D_{H}^{-}(x) \subset K_{2}+K_{1}+L_{2}+L_{1} \subset T^{*}$ (or else $K_{2}$ could have been made larger) so $W \subset D^{+}(x)$. Likewise strict inequality in (ii) implies $W \subset D^{2}(y)$. Now $d \leq|S|-33$ means

$$
|W| \leq b_{1}+b_{2}-k-l-32-|Z|
$$

so we can partition $W$ as $X \cup Y$ with

$$
|X| \leq b_{1}-k-16 \text { and }|Y| \leq b_{2}-l-16 \text {. }
$$

If $X \neq \varnothing$ then inequality (i) is strict, so $X \subset D^{+}(x)$, and likewise $Y \subset D^{2}(y)$ if $Y \neq \varnothing$. Since $|Z|+6 \leq 16$, this is the partition of $W$ we seek.

Case (b): $d_{H}^{-}(x) \leq 5$ and $d_{H}^{1}(y) \geq 6$, or $d_{H}^{-}(x) \geq 6$ and $d_{H}^{1}(y) \leq 5$. We prove this case on the assumption that the first pair of inequalities hold. It will then be clear how the proof should be modified if the second pair of inequalities hold. Choose $L \subset D_{H}^{1}(y)$ as large as possible, subject to

(i) $l \leq b_{2}-16$, and

(ii) $l \leq d+b_{1}+b_{2}-|S|-7$,

where $l=|L|$. Then $l \geq 2$. (However, if $P_{2}=P(q, \ldots)$ has order 1 or 2 , so $B_{1}$ and $B_{2}$ are adjacent in $C$ or separated by one edge, we choose $L \subset D_{H}^{1}(y) \cap D_{H}^{+}(x)$, again subject to (i) and (ii). Note $l \geq 1$ since $d_{H}^{+}(x) \geq|H|-5$, and in this case alone we allow $l=1$.)

Let $P^{*}=P(q-1, \ldots, l-1)$, or $P^{*}=P(l-1)$ if $\left|P_{2}\right|=1$ or 2 . We choose $K \subset D_{H}^{+}(x)$ and $T^{*}$ as follows. If $P_{2}$ has order 1 or 2 we put $T^{*}=K=L$. It is possible here that $l=1$ and $T^{*}$ is a single vertex. Otherwise choose $K \subset D_{H}^{+}(x)-L$ with $|K|=q+2$. This is possible because $|H|=n-|S|$ and $n \geq d+b_{1}+b_{2}+q$, so by (ii)

$$
d_{H}^{+}(x) \geq|H|-5 \geq d+b_{1}+b_{2}+q-|S|-5 \geq l+q+2 .
$$

If $P^{*}$ has two blocks, that is $P^{*}=P(q-1, l-1)$, put $T^{*}=K \cup L$, so $\left|T^{*}\right|=\left|P^{*}\right|+3$. Otherwise choose $T^{*} \subset H$ with $K \subset T^{*}, L \subset T^{*}$ and $\left|T^{*}\right|=\left|P^{*}\right|+2$; again this is possible because by (ii),

$$
\left|P^{*}\right|+2=n-d-b_{1}-b_{2}+l+1 \leq n-|S|-6<|H| .
$$

Using Theorems 3-5 we find $P^{*}$ in $T^{*}$ beginning in $K$ and ending in $L$. Because $K \subset D_{H}^{+}(x)$ and $L \subset D_{H}^{1}(y)$ we then have $P(q, \ldots, l)$ from $x$ to $y$, and to complete $C$ we will find $P\left(b_{1}, m, \ldots, b_{2}-l\right)$ in $T-P^{*}$ beginning at $x$ and ending at $y$. Put $T-P^{*}=S \cup W \cup Z^{\prime}$, where $W=T-T^{*}-S-D_{H}^{-}(x)$ and $Z^{\prime}=T^{*}+D_{H}^{-}(x)-P^{*}$. Note $\left|Z^{\prime}\right| \leq\left|D_{H}^{-}(x)\right|+\left|T^{*}-P^{*}\right| \leq 8$. Once more we are home by Lemma 12 if we partition $W \cup Z^{\prime}$ as $X \cup Y \cup Z$ with $X \subset D^{+}(x), Y \subset D^{2}(y), b_{1} \geq|X|+|Z|+6$ and $b_{2}-l \geq|Y|+|Z|+6$.

Now $|W|+\left|Z^{\prime}\right|=n-\left|P^{*}\right|-|S|=d+b_{1}+b_{2}+1-l-|S|$. If equality holds in (ii), then $|W|+\left|Z^{\prime}\right|=8$, so it is satisfactory to put $Z=W \cup Z^{\prime}$ and $X=Y=\varnothing$, because $b_{1} \geq 18$ and $b_{2}-l \geq 16$. If inequality is strict in (ii), put $Z=Z^{\prime}$ and 
$W=X \cup Y$, where $|X| \leq b_{1}-16$ and $|Y| \leq b_{2}-l-16$. This is possible because $|W| \leq b_{1}+b_{2}-l-32$. Observe that $|Z|+6 \leq 16$ since $\left|Z^{\prime}\right| \leq 8$, and that $X \subset W \subset D^{+}(x)$ by definition of $W$. Finally, if $Y \neq \varnothing$ then inequality is strict in (i) also, and hence $\left(D_{H}^{1}(y) \cap D_{H}^{+}(x)\right) \subset L \subset T^{*}$ (otherwise $L$ would have been chosen larger). But $W \subset\left(T-T^{*}\right) \cap D_{H}^{+}(x)$ so $Y \subset W \subset D^{2}(y)$, as desired.

Case (c): $d_{H}^{-}(x) \leq 5, d_{H}^{1}(y) \leq 5$. Let $T^{*}=D_{H}^{+}(x) \cap D_{H}^{2}(y)$; then $\left|T^{*}\right| \geq|H|-10$. If $P_{2}=P(q, \ldots)=P(q, \ldots, r)$ has order at least 3 , let $P^{*}=P(q-1, \ldots, r-1)$ (or if $P_{2}=P(q)$ put $\left.P^{*}=P(q-2)\right)$. Then $\left|P^{*}\right| \geq 1$, and

$$
\left|P^{*}\right|=\left|P_{2}\right|-2=n-d-b_{1}-b_{2}-1 \leq n-|S|-11=|H|-11 \leq\left|T^{*}\right|-1 .
$$

Hence by Corollary 2 we may find $P^{*}$ in $T^{*}$, giving $P_{2}$ between $x$ and $y$. If $P_{2}$ has order 2 we already have $P_{2}$ between $x$ and $y$, since $x y \in S$; put $P^{*}=\varnothing$ in this case. Thus if $\left|P_{2}\right| \geq 2$ we need only find $P\left(b_{1}, m, \ldots, b_{2}\right)$ from $x$ to $y$ in $T-P^{*}$. If $P_{2}$ is a single vertex, it is enough to find $P\left(b_{1}, m, \ldots, b_{2}-1\right)$ in $T$ from $x$ to $y$, since $x y \in S$; put $P^{*}=\varnothing$ in this case also. Let $b=b_{2}$ if $\left|P_{2}\right| \geq 2$ and $b=b_{2}-1$ if $\left|P_{2}\right|=1$.

Let $Z=D_{H}^{-}(x) \cup D_{H}^{1}(y)$; then $|Z| \leq 10$. Let $W=T-Z-S-P^{*}$. We must find $P\left(b_{1}, m, \ldots, b\right)$ in $T-P^{*}$ from $x$ to $y$. Since $T-P^{*}=W \cup Z \cup S$, we may apply Lemma 12 provided we partition $W$ as $X \cup Y$, where $b_{1} \geq|X|+|Z|+6$ and $b \geq|Y|+|Z|+6$. (Observe that, by definition, $W \subset D_{H}^{+}(x) \cap D_{H}^{2}(y)$, so $X \subset D^{+}(x)$ and $Y \subset D^{2}(y)$.) To do this, just choose $X$ and $Y$ with $|X| \leq b_{1}-16$ and $|Y| \leq b-16$. This is possible since

$$
|W|+|Z|=d+b_{1}+b+1-|S| \leq b_{1}+b-32 .
$$

From Lemmas 9, 11 and 13 follows the main result.

THEOREM 14. Let $C$ be a non-strongly oriented cycle of order $n$. Let $T$ be a tournament of order $n$. If $T$ contains a transitive subtournament of order 129 (in particular if $n \geq 2^{128}$ ), then $T$ contains $C$.

ProOF. Label the edges of $C$ consecutively $e(0), e(1), \ldots, e(n-1)$ so that $e(115)$ and $e(116)$ are in different blocks. Let $S$ be a maximal transitive subtournament of $T$ with $|S| \geq 129$, and let $m=|S|-11 \geq 118$. For $0 \leq j \leq 115$ we may assume that $e(j)$ and $e(m+j)$ are in different blocks (addition is $\bmod n)$, else as $m+j \geq 118$ they are both in a block of length at least $n-|S|+11$, whence by Lemma $9 T$ contains $C$.

Now suppose that for some $t, 0 \leq t \leq 4$, the edges $\{e(23 t+j) ; 0 \leq j \leq 21\}$ are in the same block $B_{1}$ of length $b_{1} \geq 22$ and the edges $\{e(23 t+j+m) ; 0 \leq j \leq 21\}$ are in the same block $B_{2}$ of length $b_{2} \geq 22$. Then $B_{1} \neq B_{2}$, and if the distance between $B_{1}$ and $B_{2}$ (measured along the path of $C$ containing $e(115)$ ) is $d$ we have

$$
d \leq 23 t+m-1-(23 t+21) \leq|S|-33
$$

and $d+b_{1}+b_{2} \geq 23 t+m+21-(23 t-1)=|S|+11$. Then by Lemma $13 C$ is in $T$.

Otherwise, either there are at least three values of $t, 0 \leq t \leq 4$, for which the set $\{e(23 t+j) ; 0 \leq j \leq 21\}$ contains edges from different blocks, or there are at least three values of $t$ for which the set $\{e(23 t+j+m) ; 0 \leq j \leq 21\}$ contains edges from different blocks. Construct a set $E$ of edges as follows. In the first case, let $e(k)$ be 
the first edge in a different block from $e(0)$, and put $E=\{e(k), \ldots, e(115)\}$. In the second let $k$ be the largest integer with $k \leq 112+m$ and with $e(k)$ and $e(113+m)$ in different blocks, and put $E=\{e(116), \ldots, e(k)\}$. Then $50 \leq|E| \leq|S|-14$, and $E$ satisfies the conditions of Lemma 11 , so $T$ contains $C$.

COROLlary 15. Let $T$ be a tournament of order $n \geq 2^{128}+1$. Then $T$ is pancyclic; that is, $T$ contains every non-strongly oriented cycle $C$ with $3 \leq|C| \leq n$.

Proof. If $|C| \leq 13$, then $C \subset T T_{13} \subset T$. If $14 \leq|C| \leq n-2$, then $C \subset T$ by Corollary 6. Otherwise $n-1 \leq|C| \leq n$ and $C \subset T$ by Theorem 14 .

ACKNOWLEDGEMENT. The author wishes to thank a referee for his careful reading and many thoughtful comments.

\section{REFERENCES}

1. B. Alspach and M. Rosenfeld, Realisation of certain generalised paths in tournaments, Discrete Math. 34 (1981), 199-202.

2. A. Benhocine and A. P. Wojda, On the existence of a specified cycle in a tournament, J. Graph Theory 7 (1983), 469-474.

3. B. Bollobás, Extremal graph theory, Academic Press, New York, 1978.

4. R. Forcade, Parity of paths and circuits in tournaments, Discrete Math. 6 (1973), 115-118.

5. B. Grünbaum, Antidirected hamiltonian paths in tournaments, J. Combin. Theory Ser. B 11 (1971), 249-257.

6. M. C. Heydemann, D. Sotteau and C. Thomassen, Orientations of hamiltonian cycles in digraphs (to appear).

7. K. B. Reid and N. C. Wormald, Embedding oriented n-trees in tournaments (to appear).

8. M. Rosenfeld, Antidirected hamiltonian paths in tournaments, J. Combin. Theory Ser. B 12 (1972), 93-99.

9. (1974), 234-242.

10. H. J. Straight, The existence of certain types of semi-walks in tournaments (Proc. XI Southeastern Conference on Combinatorics, Graph Theory and Computing), Congress. Numer. 29 (1980), 901-908.

11. C. Thomassen, Antidirected hamiltonian circuits and paths in tournaments, Math. Ann. 201 (1973), 231-238.

Department of Mathematics, Louisiana State University, Baton Rouge, LOUISIANA 70803

St. John'S COllege, CAMBridge, ENGland land

Current address: Department of Mathematics, University of Exeter, Exeter, EX4 4QE, Eng- 